\title{
Problèmes posés par la recherche et le dosage des matières grasses végétales (MGV) dans le chocolat
}

Oléagineux, Corps Gras, Lipides. Volume 10, Numéro 4, 309-14, JUILLET-AOÛT 2003, Problématiques actuelles dans le domaine de l'analyse des oléagineux et des corps gras

Auteur(s) : Henri CHAVERON, Professeur honoraire des Universités, Expert Judiciaire près la Cour d'Appel de Paris 48, rue de la Fontaine 3 Etots 60190 Cernoy .

Résumé : La nouvelle directive 2000\\36\\CE du Parlement européen et du Conseil autorise l'emploi de certaines matières grasses végétales (MGV), à une teneur maximale de $5 \%$ dans le chocolat. Dans ce nouveau contexte, sont ici examinées les possibilités analytiques de vérification de la bonne appréciation de cette directive. Les notions de détection et de quantification sont précisées. Les rôles respectifs joués par ces deux approches analytiques dans la recherche de la conformité des produits finis sont définis. Les recherches analytiques effectuées au niveau européen, depuis plus d'un quart de siècle, tant dans le cadre de la Commission que dans celui de I'Industrie du chocolat, sont examinées et critiquées. L'attention est finalement attirée sur la complexité des problèmes de détection des matières végétales autorisées (MGV), dans un environnement lipidique très diversifié, complexité qui classe ces problèmes dans la catégorie de ceux "auxquels aucune solution satisfaisante n'a encore pu être apportée ».

Mots-clés : chocolat, beurre de cacao ( $B C)$, matières grasses végétales (MGV), détection dosage, directive 2000\\36\\CE

\section{ARTICLE}

\section{Auteur(s) : Henri CHAVERON}

Professeur honoraire des Universités, Expert Judiciaire près la Cour d'Appel de Paris 48, rue de la Fontaine 3 Etots 60190 Cernoy

\section{Introduction}

Dès leur entrée dans le marché commun, les chocolatiers britanniques, danois et irlandais, qui employaient depuis longtemps diverses matières grasses végétales (MGV), ont souhaité que cette pratique s'étende à l'ensemble de la communauté.

Deux conceptions se sont opposées dès le début des discussions sur cette question au niveau de la CEE : I'une "traditionaliste ", basée sur le principe de l'authenticité des matières premières, en l'occurrence du beurre de cacao (BC), l'autre "progressiste », reposant sur une argumentation " économico-technologique ".

Pour les "traditionalistes ", le BC est la seule matière grasse permettant d'obtenir du chocolat. Son remplacement, même partiel, ne peut que compromettre la qualité de cet aliment. 
Cette attitude est jugée peu favorable à " l'évolution technico-économique " par les " progressistes » qui préfèrent se libérer des contraintes résultant des propriétés très marquées du beurre de cacao et produire le chocolat dans les meilleures conditions de rentabilité. Un quart de siècle de pourparlers, pour ne pas dire d'affrontements de conceptions, s'est terminé, en 2000, par l'adoption d'un compromis. Six MGV ${ }^{1}$ d'origine tropicale pourront, dorénavant, être utilisées dans la fabrication du chocolat ${ }^{2}$. Le problème posé à l'analyste est de vérifier dans le chocolat :

- que les quantités de MGV autorisées ne dépassent pas les $5 \%$ fixés comme valeur maximale par la directive (quantification),

- qu'aucune autre MGV n'est présente (détection). Mais aucune méthode officielle n'est mise à sa disposition.

${ }^{1}$ Graisses de tengkawang et de sal, Beurres de karité et de Kokum, Huiles de Palme et de Mangue.

${ }^{2}$ Le chocolat est fabriqué en utilisant de la masse de cacao (broyat de fèves de cacao torréfiées contenant environ $50 \%$ de beurre de cacao) du sucre et du beurre de cacao (BC). C'est ce BC ajouté qui pourra être remplacé par des MGV

\section{Les MGV pouvant être utilisées en chocolaterie}

Elles se répartissent en trois catégories principales :

1. Les MGV dans la composition desquelles entrent principalement les acides stéarique $\left(\mathrm{S}_{\mathrm{t}}\right)$, oléique $(O)$ et palmitique (P). Ces MGV présentent une composition triglycéridique (TG) relativement simple comme celle du beurre de cacao. Les triglycérides monoinsaturés symétriques $P O P, \operatorname{POS}_{t}, S_{t} O S_{t}$, principaux glycérides du BC caractérisent ces MGV qui se distinguent entre elles par la teneur globale en triglycérides monoinsaturés et par leur proportion relative en $\mathrm{S}_{\mathrm{t}} \mathrm{OS} \mathrm{S}_{\mathrm{t}}$ et POP.

2. Les MGV à teneurs élevées en A.G. insaturés trans proviennent d'huiles durcies par hydrogénation. La composition triglycéridique de ces MGV est d'une très grande complexité du fait de la présence de nombreux isomères de position cis et trans des A.G. insaturés.

3. Les MGV du type laurique (riches en acide laurique) présentent également une composition triglycéridique complexe.

Dans la $1^{\text {re }}$ catégorie, figurent les équivalents du BC (EBC) (figure 1) et les MGV proches de ces équivalents. Toutes ces MGV sont entièrement compatibles avec le $B C$ Dans les catégories 2 et 3 se trouvent des MGV dont la composition s'éloigne notablement de celle du BC Elles sont plus ou moins incompatibles avec le $B C$, ce qui en limite l'usage. Les MGV retenues par la directive entrent dans la catégorie 1.

\section{Détection et évaluation quantitative}

Une certaine confusion entre détection et évaluation quantitative a toujours obscurci le débat sur les MGV utilisables en chocolaterie.

La détection a pour objectif de répondre à la question «le chocolat contient-il une MGV non autorisée ? » et secondairement de préciser laquelle. 
L'objectif de l'évaluation quantitative (EQ) est de déterminer la quantité (le \%) de MGV autorisée, contenue dans un chocolat.

Dans le cadre de la nouvelle directive de I'UE, la recherche de conformité nécessite l'emploi de la détection pour vérifier que le chocolat ne renferme pas de MGV non autorisées $\left(\mathrm{MGV}_{(\mathrm{NA})}\right)$ et l'emploi de l'évaluation quantitative pour s'assurer que les MGV autorisées $\left(\operatorname{MGV}_{(\mathrm{A})}\right)$ se trouvent bien dans le chocolat à un taux au plus égal à $5 \%$.

En ce qui concerne le chocolat " pur beurre de cacao ", il continuera à être fabriqué après août 2003, date d'application en France de la nouvelle directive de I'UE, parallèlement au chocolat contenant des $\operatorname{MGV}_{(\mathrm{A})}$.

La conformité à son appellation sera déterminée par des méthodes de détection suivant exactement l'approche analytique utilisée, pour s'assurer de la conformité d'un chocolat, avant la nouvelle directive (figure 2).

\section{Evaluation quantitative des MGV dans le chocolat}

L'évaluation quantitative nécessite 2 phases :

- une phase analytique: dosage d'un marqueur (M) ou détermination de la composition triglycéridique (TG).

- une phase déductive : au cours de laquelle, par calcul à partir des informations analytiques, le chimiste tente d'évaluer la quantité de MGV dans le chocolat.

- Utilisation d'un marqueur (M)

- le marqueur peut être endogène, spécifique d'une matière grasse. Les marqueurs endogènes sont rares $^{3}$. De plus, ils sont relatifs à une catégorie de $\mathrm{MGV}^{4}$ Ils servent principalement à la détection.

L'évaluation de la quantité de MGV présente dans le chocolat à partir d'un marqueur endogène nécessite de connaître la teneur de ce marqueur dans la MGV Or, cette teneur ne peut être obtenue précisément que par analyse de la MGV utilisée. Dans la quasi-totalité des cas, le chimiste ne dispose pas de cette MGV Les MGV produites par l'industrie des corps gras n'ont pas des compositions constantes au cours du temps. Elles changent en fonction des matières premières utilisées pour les fabriquer. A cela s'ajoute l'influence des traitements physique et chimique utilisés pour l'obtention des MGV (figure 3). Dans ces conditions, les teneurs en marqueurs endogènes sont aléatoires et de ce fait, empêchent toute tentative d'évaluation quantitative de MGV dans le chocolat, lorsque la composition de celle-ci est inconnue.

- le marqueur peut être ajouté à la MGV. II est alors qualifié de marqueur exogène. Dans ce cas, la situation est, contrairement à la précédente, quasiment parfaite puisque la quantité de marqueur dans la MGV est exactement connue. 
On détermine le pourcentage de matières grasses végétales (\% MGV) dans le chocolat de la façon suivante :

$\%$ MGV=\% M chocolat) $/ \%$ M MGV) $\times 100$

Des études entreprises par CAOBISCO et le $\mathrm{FEDIOL}^{5}$ sur l'utilisation de marqueurs exogènes ont permis de mettre au point une méthode de dosage précise de marqueurs triglycériques ${ }^{6}$ dans les produits de chocolaterie. Malheureusement, cette méthode, si satisfaisante sur le plan analytique, a été considérée comme pratiquement inapplicable. En effet, comment être assuré que toutes les MGV pouvant être utilisées en chocolaterie, seront marquées et cela non seulement en Europe mais également dans le reste du monde ? De plus, la présence de marqueur peut être considéré comme indésirable ${ }^{7}$ dans le chocolat.

- Examen des variations de composition triglycéridique Toute composition triglycéridique, des matières grasses extraites d'un chocolat, qui ne s'inscrit pas dans les limites de variation généralement admises pour le beurre de cacao $(B C)$ peut faire suspecter la présence de MGV dans ce chocolat.

Les deux phases de l'évaluation quantitative sont représentées ici par :

1. la chromatographie en phase gazeuse $(\mathrm{CPG})^{8}$ des TG

2. une méthode de calcul permettant d'exploiter les résultats obtenus par CPG (encadré 1). Seule cette phase est spécifique de ces méthodes type « méthode CAOBISCO ».

Les teneurs en TG 50, 52 et 54 varient peu en fonction des différents types de BC À l'intérieur de ces faibles variations, c'est le C52 qui varie le moins. La méthode CAOBISCO est basée sur la constatation que la plupart des MGV présente une teneur en C52 plus faible que celle du BC Les variations importantes portent sur le $\mathrm{C} 50$ et le C54. Sur un diagramme, les différentes MGV sont représentées par des points de coordonnées C50/C54. La distance de ces points par rapport à la droite tracée pour les $B C$ est utilisée pour l'évaluation quantitative des MGV (figure 6). La modification ${ }^{10}$ de la composition triglycéridique de la graisse $(B C+M G V)$ extraite du chocolat par rapport à la composition moyenne du beurre de cacao en triglycérides sera d'autant plus importante que la quantité de MGV ajoutée sera plus grande (et) ou que sa composition sera plus éloignée de celle du $\mathrm{BC}$

A l'inverse, plus la composition en TG d'une MGV sera proche de celle du BC, moins la modification triglycéridique sera sensible et plus cette MGV sera difficile à évaluer quantitativement et même, à la limite, à détecter.

Ce qui signifie pratiquement que certaines matières grasses naturelles comme celles de l'illipé de Bornéo ou celles obtenues par voies chimique ou biotechnologique ne pourront pas être quantifiées ni même détectées à la dose d'incorporation de $5 \%$ par rapport au produit fini soit environ $15 \%$ par rapport aux matières grasses totales du chocolat.

${ }^{3}$ Les progrès des techniques analytiques permettent de mettre en évidence de nouveaux marqueurs et par voie de conséquence d'augmenter les possibilités de détection. ${ }^{4}$ Le fait que les rares marqueurs existants ne concernent que des catégories particulières MGV est un 
"vice rédhibitoire» pour la méthode. Si par exemple en utilisant le marqueur AG trans, on détermine dans le chocolat et dans la graisse hydrogénée utilisée le \% de trans, on pourra évaluer avec précision la quantité de graisse hydrogénée ajoutée. Mais si cette graisse a été mélangée avec une MGV avant l'incorporation au chocolat (ce qui se passe fréquemment), l'évaluation ne concernera que la graisse hydrogénée et non pas la MG totale (graisse hydrogénée + autre MGV) additionnée.

${ }^{5}$ CAOBISCO : Association des Industries de la Chocolaterie, Biscuiterie-Biscotterie et Confiserie de I'UEFEDIOL: Fédération Européenne des Fabricants de Matières Grasses. ${ }^{6}$ Les experts ont porté leur choix sur 2 TG synthétiques le triheptanoïde et le trinonanoïde.

${ }^{7}$ Bien que les marqueurs soient de même nature (TG) que les MGV qu'ils ont pour rôle de quantifier et qu'ils soient obtenus par des procédés qui peuvent être utilisés pour la fabrication des MGV. De plus, les quantités utilisées sont très faibles. Enfin, les marqueurs exogènes ont été utilisés à plusieurs reprises dans d'autres domaines alimentaires.

${ }^{8}$ Les conditions adoptées pour la CPG sont telles que les triglycérides sont séparées en fonction du nombre d'atomes de carbone (N.C.). Le pourcentage de chacun des triglycérides C50, C52, C54 est calculé par rapport à la $\Sigma \mathrm{C} 50+\mathrm{C} 52+\mathrm{C} 54$.

${ }^{9}$ La méthode CAOBISCO est décrite dans l'article : Influence des matières grasses polyinsaturées sur la détection et le dosage des MGV dans le chocolat. H. CHAVERON, C. VERDOIA, M. MEILI Ann Fals.Exp. Chim., 1984, 883, 571.

${ }^{10}$ Cette modification peut avoir d'autres origines que l'addition de MGV. Des matières grasses provenant de matières comestibles ajoutées au chocolat peuvent modifier la composition des TG des MG. totales. En particulier, en présence d'huile d'amandes ou de noisettes, il sera nécessaire de tracer un nouveau diagramme C50 C54 à partir des TG monoinsaturés symétriques séparés de la MG extraite des chocolats par la technique mise au point par H. CHAVERON et COLL. (voir note 9).

\section{Détection des MGV}

La détection ne comprend qu'une phase, à savoir la phase analytique. Elle n'est donc fonction que de la qualité de la méthode utilisée en particulier de sa sensibilité. Mettre en évidence dans le chocolat le marqueur endogène d'une $\mathrm{MGV}^{11}$ est la preuve de la présence de cette graisse dans le chocolat. La détection des $\mathrm{MGV}_{(\mathrm{NA})}$ exige une connaissance approfondie de leur composition. Ceci afin de trouver chez elles des caractéristiques qui les différencient des $\mathrm{MGV}_{(\mathrm{A})}$ et du BC. Avant l'adoption de la liste restreinte par le Parlement européen, il avait été proposé une liste de 28 MGV d'origine tropicale. Liste qui elle-même ne représente qu'une partie de l'ensemble des MGV utilisables en chocolaterie. Les connaissances limitées, dont on dispose actuellement sur ces graisses, permettent toutefois de prédire qu'un nombre très faible d'entre elles, du fait de la rareté des marqueurs endogènes, pourra être détecté dans la phase lipidique complexe d'un chocolat. Ce qui signifie que dans certains cas (relativement rares), il sera possible de conclure à la non-conformité d'un chocolat mais jamais à sa conformité (figure 2).

Dans le cadre de la nouvelle directive de I'UE, la mise en évidence dans un chocolat d'une MGV interdite, suffit pour conclure à sa non-conformité (comme dans le cas de la précédente réglementation). 
Le problème de la détection des MGV interdites est généralement minimisé si non occulté alors qu'il est, sur le plan de la conformité, aussi important que celui de l'évaluation quantitative des MGV $_{(\mathrm{A})}$ autorisées (figure 2).

${ }^{11}$ Par exemple, la mise en évidence d'acide élaïdique dans le chocolat amène à suspecter la présence d'une graisse hydrogénée.

\section{Travaux effectués au niveau de l'UE}

Les travaux des experts de CAOBISCO ont permis de mettre au point ou de sélectionner, après une étude exhaustive de toutes les possibilités, des méthodes à la fois pour la détermination des caractéristiques des "MGV » et pour l'évaluation quantitative de ces MGV dans le chocolat (publication interne en 1982 de la méthode CAOBISCO). Depuis, des progrès ont été réalisés dans le domaine de l'analyse qui n'ont profité, très modestement d'ailleurs, qu'à la détection puisque, les possibilités de l'évaluation quantitative sont peu influencées par la qualité des méthodes analytiques. Ce qui explique que malgré l'évolution de celles-ci, la détermination du pourcentage de MGV dans le chocolat n'ait pas évolué depuis les travaux des experts de CAOBISCO. Dans le même temps, d'importants progrès ont été faits dans le domaine de l'obtention des MGV, certes par voie chimique, mais également par voie biotechnologique. Ce qui a pour conséquence une augmentation potentielle du nombre de MGV proches du BC, donc non dosables et économiquement intéressantes (figure 1)

Une étude en collaboration pilotée par le JRC ${ }^{12}$ d'Ispra (Italie) a été entreprise entre 1997 et 1999 avec l'aide de 11 participants européens.

La majorité des techniques testées concernaient la détection, notamment celle des matières grasses raffinées et hydrogénées.

Sur la base de variations de composition triglycéridique, des essais ont été faits en utilisant I'H.P.L.C. et H.R.G.C ${ }^{13}$. D'après leurs auteurs, l'utilisation de ces techniques analytiques performantes n'améliore pas l'évaluation quantitative des MGV obtenue par l'analyse des TG en fonction du NC (figure 5)

${ }^{12}$ JRC (Joint research Centre) de la Commission Européenne en 1997.

${ }^{13}$ HPLC : Chromatographie Liquide Haute Performance

HRGC : Chromatographie en Phase Gazeuse Haute résolution

\section{Conclusion}

Pour le contrôle des MGV dans le chocolat, un certain "flou artistique " a été "savamment " entretenu, dans les négociations, entre détection et évaluation quantitative. Ces deux notions aux finalités différentes doivent pourtant être bien distinguées.

II ne peut exister de solution analytique globale satisfaisante au problème de la détection des $M_{G V} V_{(N A)}$ non autorisées dans le chocolat. En effet, il n'est pas possible de trouver une caractéristique commune (molécule spécifique, composition triglycéridique) à l'ensemble des MGV non autorisées, qui puisse les distinguer du beurre de cacao et des MGV autorisées. Le problème de la détection ne 
peut être abordé que cas par cas. De nombreuses graisses d'origines tropicales, et proches du BC et des MGV autorisées, ne pourront pas être mises en évidence dans le chocolat.

Quant au dosage des MGV (détermination du pourcentage dans le chocolat), il va de l'estimation acceptable (fraction de palme) à l'impossibilité d'évaluer (graisse d'illipé de Bornéo, E.BC hémisynthétiques).

Ce qui revient à dire que quelques fraudes grossières ou maladroites pourront éventuellement être mises en évidence alors que les autres, plus élaborées, échapperont à la vérification de la conformité (figure 2).

Ainsi donc, aucune méthode (ou faisceau de méthode) ne peut être proposée, en toute honnêteté scientifique, comme moyen de vérifier la bonne application de la nouvelle "directive chocolat». Malgré cela, les deux dernières décennies de recherche sur la détection et l'évaluation quantitative des MGV ont été jalonnées d'annonces de mises au point de méthodes fiables dans ce domaine. Aucune d'entre elles n'a encore vu le jour. $n$ 


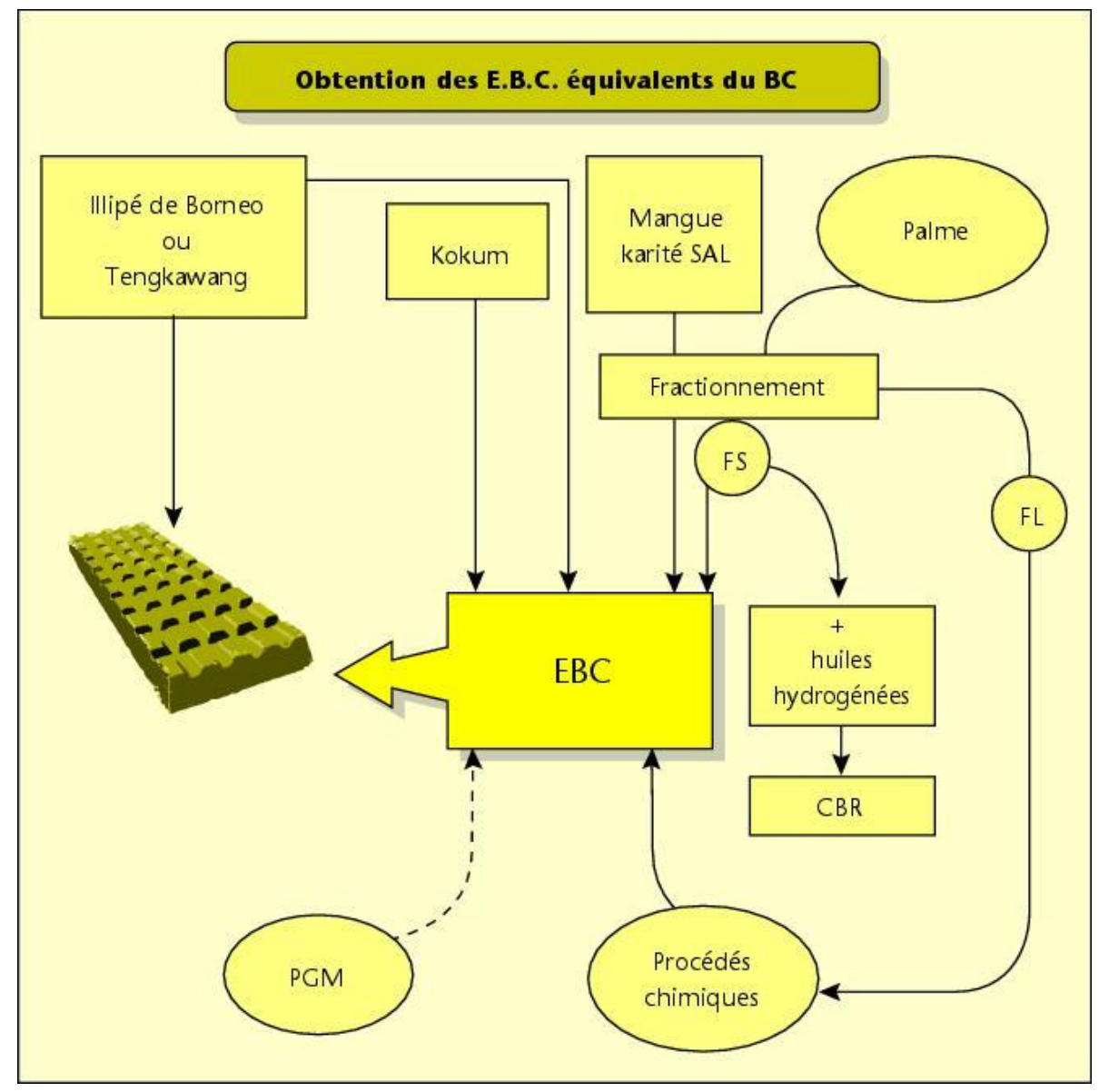

Figure 1. Obtention des EBC (équivalents du beurre de cacao).

Seul parmi les 6 MG retenues par I'UE, I'illipé de Bornéo ou Tengkawang peut être considéré comme un EBC du fait de sa composition très proche de celle du BC. Il peut être utilisé directement dans la fabrication du chocolat sans modifier de façon perceptible les propriétés de celui-ci. Les autres MGV ont des compositions en triglycérides mono insaturés symétriques, assez éloignées de celles $d u B C$. Stricto sensu, elles ne peuvent pas être considérées comme des EBC. Mais mélangés dans des proportions adéquates elles permettent de les obtenir. L'huile de palme dans le groupe des MGV de I'UE a une situation toute particulière. Elle est obtenue à partir d'une plante cultivée à grande échelle. Sa disponibilité est bonne et son coût bas contrairement aux autres MGV de la directive européenne. Les applications sont nombreuses. Après fractionnement, sa partie solide (FS) peut participer à l'élaboration des EBC. Elle peut également être utilisée seule ou en mélange avec des huiles hydrogénées pour donner les cocoa butter replacers (CBR). Enfin les fractions liquides ( $F L$ ) peuvent servir à la fabrication par voie chimique d'EBC hémisynthétiques lesquels se distinguent des EBC naturels de deux manières importantes :

1) il est possible d'obtenir pour eux une composition identique à celle du BC leur permettant d'avoir ainsi les mêmes propriétés que celui-ci

2) ils peuvent en toute légalité et dans des conditions de rentabilité maximum, permettre la fabrication de chocolat conservant toutes leurs propriétés. Hors légalité certains seraient peut être tentés du fait de l'impossibilité de dosage de ce type d'EBC, de dépasser la limite réglementaire de $5 \%$. Dans ces conditions si l'avenir des EBC hémisynthétiques est assuré celui des EBC naturels est 
compromis, leur coût étant dissuasif. Les procédés enzymatiques sont eux expressément exclus dans la directive. Par contre rien n'interdit les produits issus de plantes génétiquement modifiées (PGM) (le palmier pourrait être envisagé à plus ou moins long terme)

II ne semble pas non plus y avoir de place dans la directive pour les EBC obtenus à partir de microorganismes.

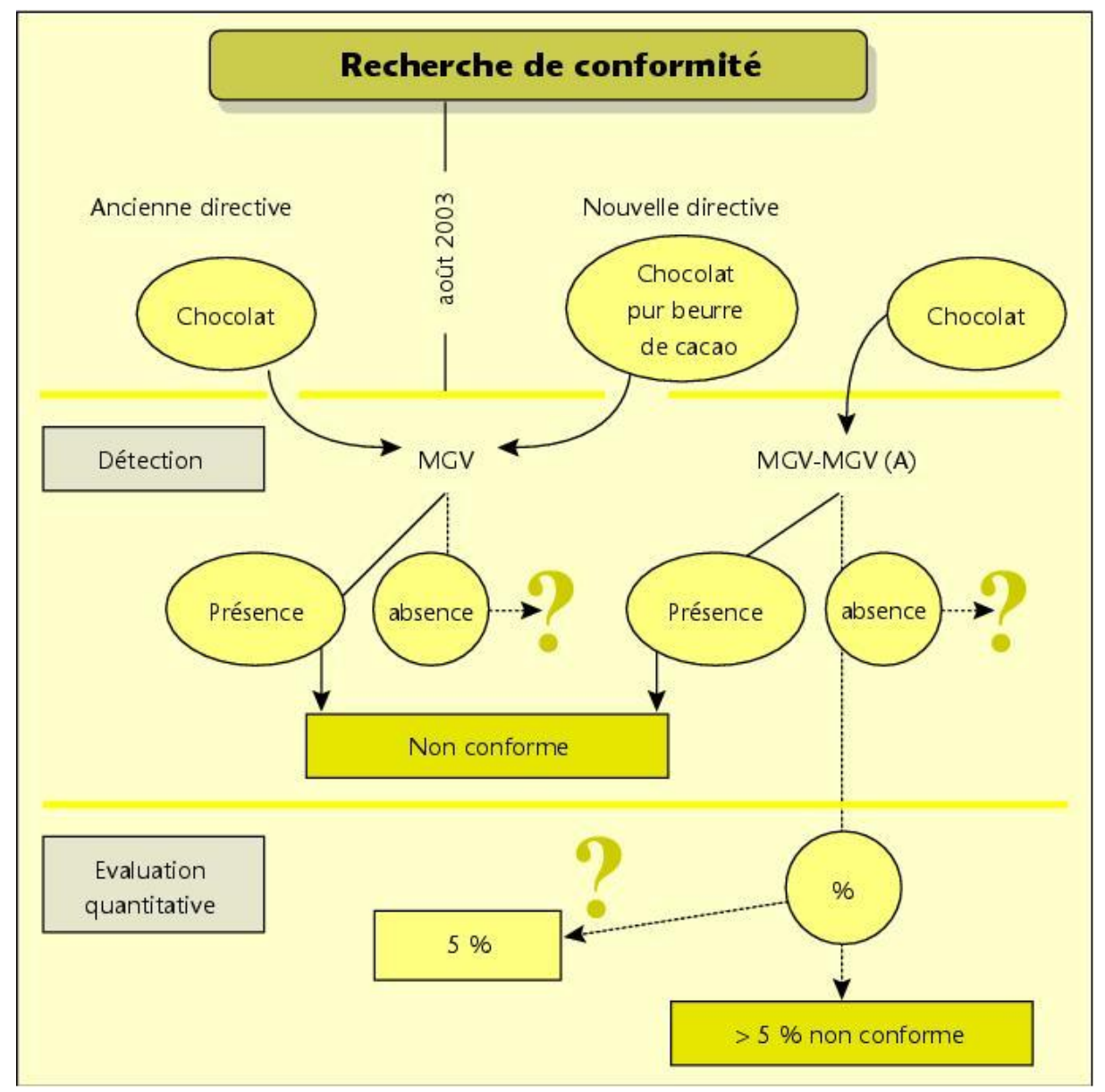

Figure 2. Détection et évaluation quantitative des MGV dans le chocolat, recherche de conformité. Si aucune MGV non autorisée (MGV $\left.{ }_{(\mathrm{NA})}\right)$ n'est mise en évidence dans le chocolat, il n'est pas possible pour autant de conclure à la conformité de celui-ci aussi bien dans le cadre de l'ancienne directive que dans celui de la nouvelle. 


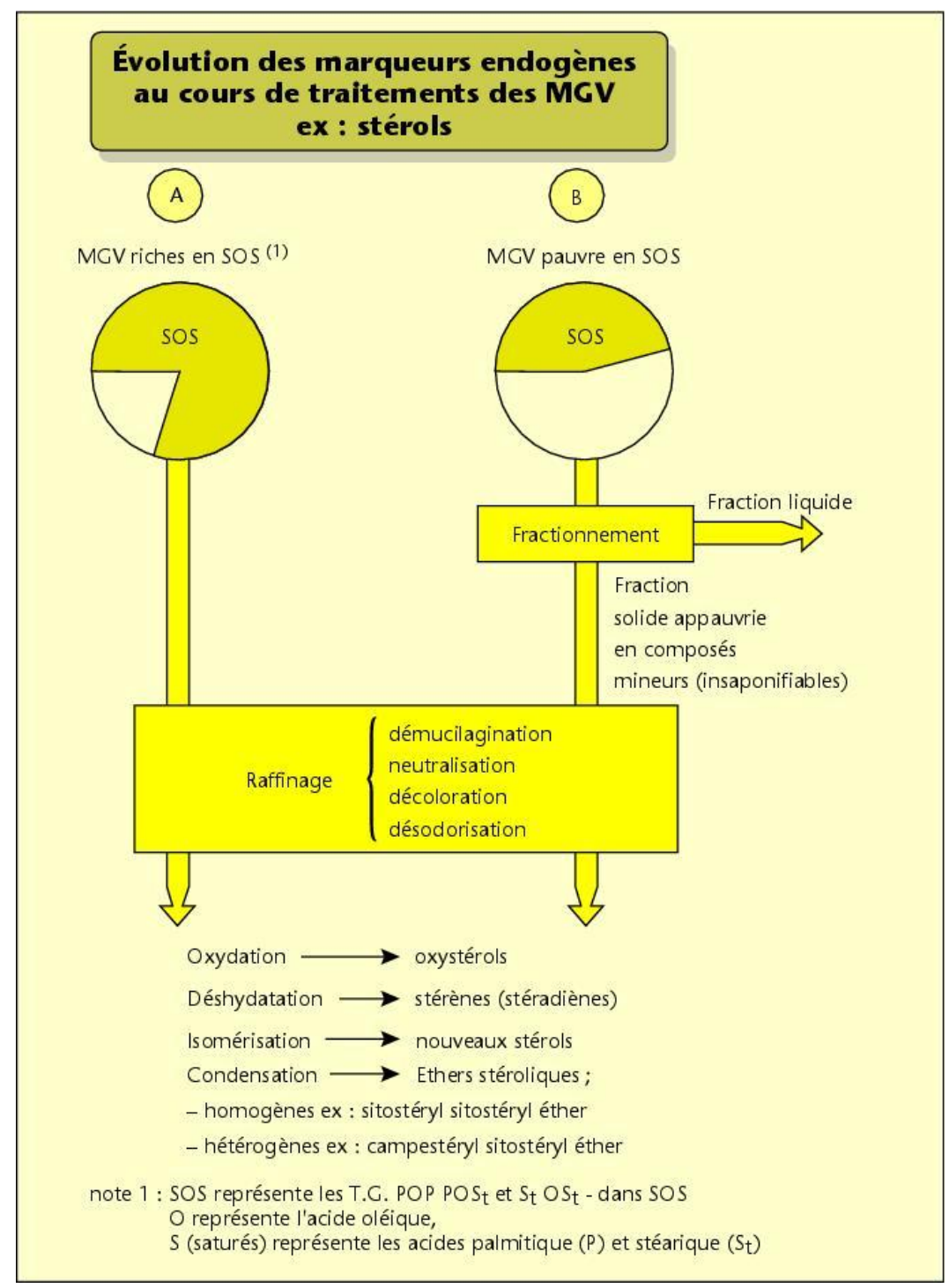

Figure 3. Evolution des marqueurs endogènes au cours des traitements des MGV (exemple : stérols). Certaines MGV (palme, sal, karité, mangue) pouvant être utilisées dans la fabrication du chocolat, nécessitent un fractionnement pour enrichir leurs fractions SOS (triglycérides mono insaturés symétriques). D'autres comme l'illipé de Bornéo très riches en SOS peuvent être utilisés sans fractionnements.

L'évaluation quantitative des $M G V_{A}$ est théoriquement possible à partir du dosage d'un marqueur endogène qui leur serait à la fois commun et spécifique. Mais elle n'est pratiquement pas réalisable. II faudrait tout d'abord disposer de données sur la dispersion biologique (variations naturelles des teneurs) alors que l'origine botanique n'est généralement pas acquise. Par la suite une cascade d'indéterminations prend naissance au moment des traitements des MGV. En particulier lors du fractionnement et du raffinage, les stérols et les terpènes subissent différentes modifications - listées en bas de la figure - qui sont à l'origine de plus ou moins grandes variations de leurs teneurs. Ce qui rend toute évaluation quantitative des MGV à partir de tels marqueurs endogènes illusoire. 


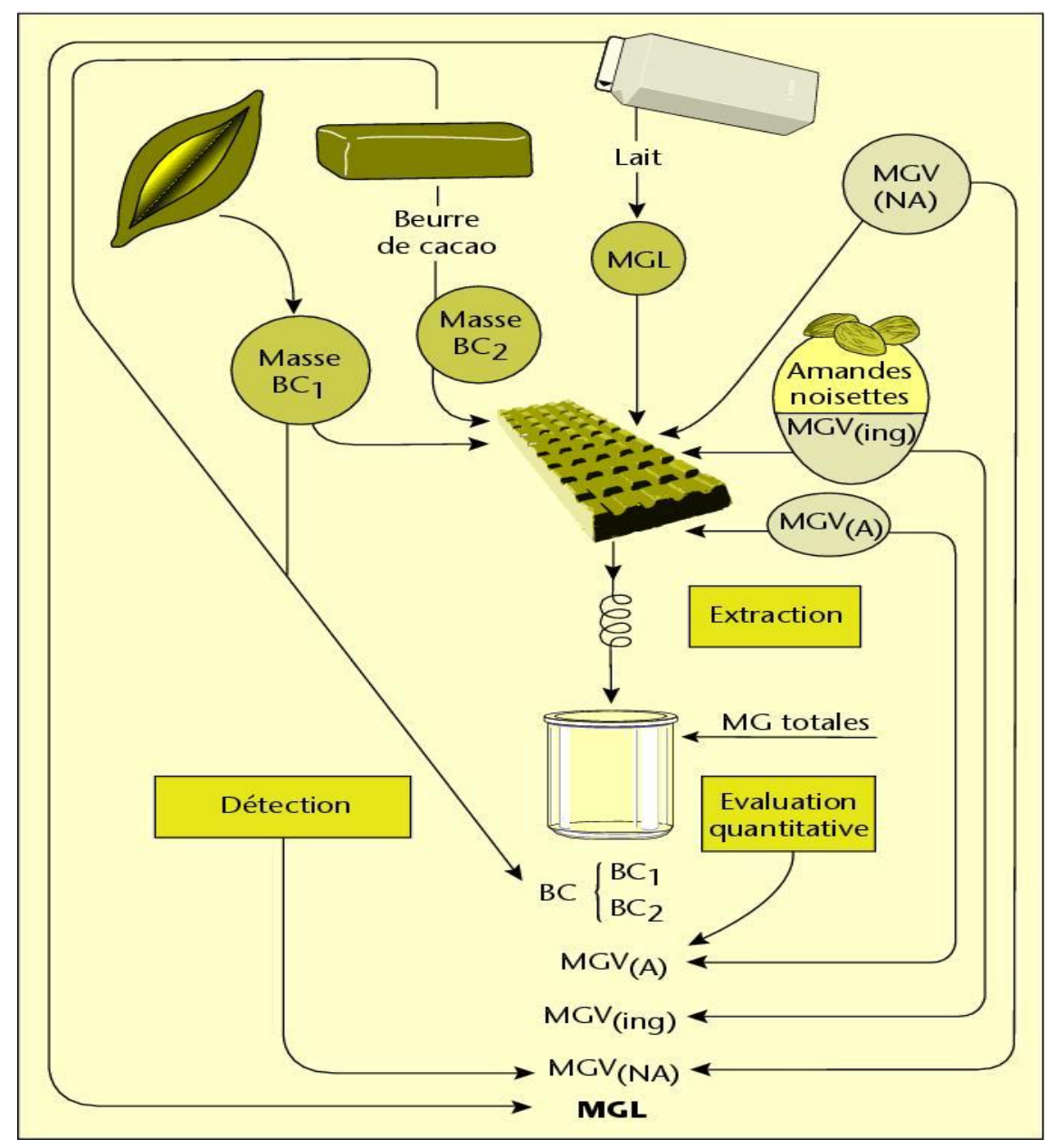

Figure 4. Contexte lipidique du chocolat et analyse des MGV.

Les matières grasses pouvant légalement se trouver dans le chocolat comprennent outre le beurre de cacao $\left(B C_{1}\right)$ provenant de la masse de cacao (note 2) et celui qui a été ajouté $\left(B C_{2}\right)$-Les 6 MGV autorisées (MGV (A).

Karité, Kolium, Sal, Illipé de Bornéo (ou Tenglsawang), ainsi que toutes les MGV provenant d'ingrédients comestibles utilisés dans la fabrication du chocolat comme par exemple les amandes et les noisettes pour ne citer que les plus importantes (il est à noter que les huiles de noisette et d'amandes ou autres huiles ou graisses provenant d'ingrédients oléagineux ne sont pas autorisées en tant que telles).

S'ajoute à cette liste un ensemble légalement ambigu comprenant des MGV obtenues par voie chimique ${ }^{14}$, notamment les E.BC hémisynthétiques.

Ces MGV n'ont pas fait, dans la directive, l'objet d'une interdiction explicite. Seules, en effet, les modifications de la structure triglycéridique de manière enzymatique sont expressément interdites. Dans le cas des chocolats au lait, la présente de Matières Grasses de Lait (MGL), seule matière grasse animale autorisée dans le chocolat, complique l'évaluation quantitative des $M G V_{(\mathrm{A})}$. Les MGV non autorisées (MGV (NA)) sont représentées par de nombreuses MGV tropicales (22 en plus des 6 retenues par le Parlement européen ont été listées dans l'annexe DGI 8767/98). Ces MGV entrent dans la catégorie 1 (voir page 310).

Les MGV (NA) comprennent également des MGV de la catégorie 2, MGV hydrogénées et de la catégorie 3, MGV lauriques. 


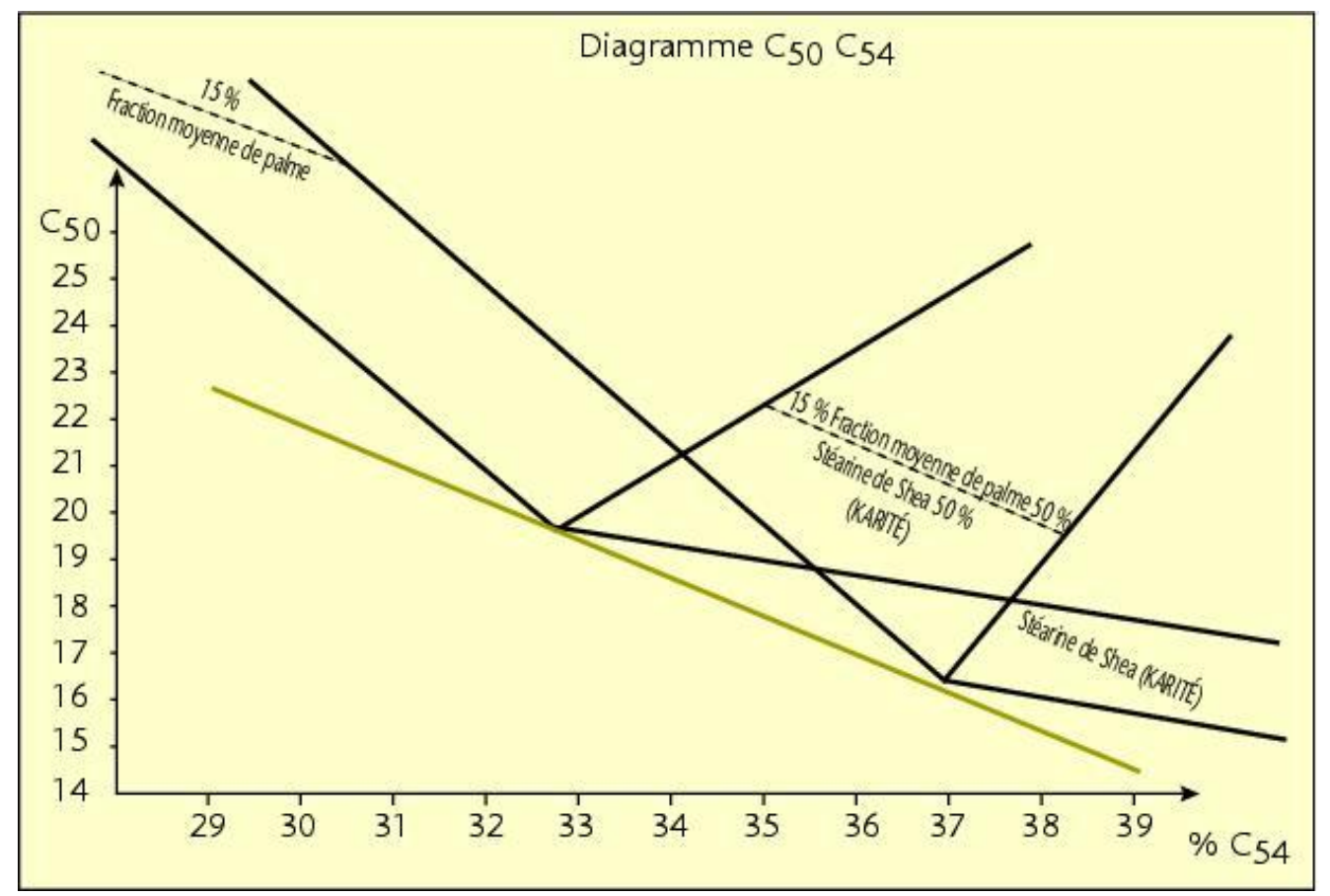

Figure 5. Les points $C_{50} C_{54}$ des différents beurres de cacao purs se situent approximativement sur une droite qui doit être établie expérimentalement par chaque laboratoire.

Cette droite partage le graphes en 2 zones : une inférieure, une supérieure. Les points représentatifs des mélanges contenant des matières grasses de remplacement se placent dans la zone supérieure. Suivant la position des points dans cette zone il est possible d'avoir une idée de la nature de la matière grasse utilisée (palme en haut à gauche, Shea en bas à droite, les mélanges des deux étant situés dans la zone médiane) et de la quantité utilisé fonction de la distance du point représentatif de la matière grasse à la droite correspondant aux beurres de cacao.

(SHEA = KARITÉ) 


\section{CALCUL DES POURCENTAGES DE MGV}

Pour un chocolat constitué d'un méiange EC + MGV en proportion $x$, il est possible d'établir les deux equations suivantes:

(1) $C_{50} B C+M G V=C_{50}^{B C}(1-x)-C_{50}{ }^{M G V} \times$

(2) $\mathrm{C}_{54} \mathrm{BC}+\mathrm{MGV}=\mathrm{C}_{54} \mathrm{BC}^{\mathrm{BC}}(1-x)+\mathrm{C}_{54}$ MGV $\mathrm{x}$

expressions dans lesquelles

$\mathrm{C}_{50} \mathrm{BC}+\mathrm{MGV} \cdot \mathrm{C}_{54} \mathrm{BC}+\mathrm{MGV} \cdot \mathrm{C}_{50}^{\mathrm{BC}} \cdot \mathrm{C}_{54} \mathrm{BC} \cdot \mathrm{C}_{50}^{\text {MGV }} \cdot \mathrm{C}_{54}^{\text {MGV }}$ sont respectivement les teneurs en TG de la MG extraite du chocolat, du BC et de la MGV

En résolvant les équations (1) et (2) à l'aide de l'équation ae la droite de régression $\% \mathrm{C}_{50}=\mathrm{a}-\mathrm{b}\left(\% \mathrm{C}_{54}\right)$ ou a et b sont respectivernent l'ordonnée ál'origine et la pente de la droite, on aboutit à l'équation:

$$
\mathrm{C}_{50} \mathrm{BC}+\mathrm{MGV}=\mathrm{a}(1-\mathrm{x})+\times\left[\mathrm{C}_{50} \mathrm{MGV}-\mathrm{bC}_{54} \mathrm{MGV}^{\mathrm{MG}}\right]-\mathrm{bC}_{54} \mathrm{BC}+\mathrm{MGV}
$$

A partir de laquelle on calcule la teneur en M.G.V. du chocolat selon Ja formule:

(3) $x=\frac{C_{50} B C-M G V-a-b C_{54} B C+M G V}{C_{50}^{M G V}-a+b C_{54} M G V}$ 100

$\mathrm{x}=$ :eneur en $\%$ de la MGV dans Jo chocolat

Encadré 1. Calcul des pourcentages de MGV. 